\title{
Observation of a vector charmoniumlike state in $e^{+} e^{-} \rightarrow D_{s}^{+} D_{s 1}(2536)^{-}+$c.c.
}

S. Jia, ${ }^{2}$ C. P. Shen $\odot,{ }^{9}$ C. Z. Yuan, ${ }^{24}$ X. L. Wang, ${ }^{9}$ I. Adachi,${ }^{15,11}$ H. Aihara, ${ }^{83}$ D. M. Asner, ${ }^{3}$ H. Atmacan, ${ }^{75}$ V. Aulchenko,, 464 R. Ayad ${ }^{78}$ V. Babu, ${ }^{8}$ I. Badhrees, ${ }^{78,33}$ A. M. Bakich, ${ }^{77}$ P. Behera, ${ }^{22}$ B. Bhuyan, ${ }^{20}$ T. Bilka, ${ }^{5}$ J. Biswal, ${ }^{30}$ A. Bobrov, ${ }^{4,64}$ G. Bonvicini, ${ }^{87}$ A. Bozek, ${ }^{60}$ M. Bračko, ${ }^{46,30}$ T. E. Browder, ${ }^{14}$ M. Campajola, ${ }^{27,55}$ L. Cao, ${ }^{31}$ D. Červenkov, ${ }^{5}$ P. Chang, ${ }^{59}$ A. Chen ${ }^{57}$ B. G. Cheon, ${ }^{13}$ K. Chilikin, ${ }^{40}$ H. E. Cho, ${ }^{13}$ K. Cho, ${ }^{34}$ S.-K. Choi, ${ }^{12}$ Y. Choi, ${ }^{76}$ D. Cinabro, ${ }^{87}$ S. Cunliffe, ${ }^{8}$ G. De Nardo, ${ }^{27,55}$ F. Di Capua, ${ }^{27,55}$ S. Di Carlo, ${ }^{38}$ Z. Doležal, ${ }^{5}$ T. V. Dong, ${ }^{9}$ S. Eidelman, ${ }^{4,64,40}$ D. Epifanov, ${ }^{4,64}$ J. E. Fast, ${ }^{66}$ T. Ferber, ${ }^{8}$ B. G. Fulsom, ${ }^{66}$ R. Garg, ${ }^{67}$ V. Gaur, ${ }^{86}$ N. Gabyshev, ${ }^{4,64}$ A. Garmash, ${ }^{4,64}$ A. Giri, ${ }^{21}$ P. Goldenzweig, ${ }^{31}$ B. Golob,${ }^{42,30}$ K. Hayasaka, ${ }^{62}$ H. Hayashii, ${ }^{56}$ W.-S. Hou, ${ }^{59}$ C.-L. Hsu ${ }^{77}$ T. Iijima, ${ }^{54,53}$ K. Inami, ${ }^{53}$ G. Inguglia, ${ }^{25}$ A. Ishikawa, ${ }^{15,11}$ R. Itoh, ${ }^{15,11}$ M. Iwasaki ${ }^{65}$ Y. Iwasaki, ${ }^{15}$ W. W. Jacobs, ${ }^{23}$ Y. Jin ${ }^{83}$ K. K. Joo, ${ }^{6}$ K. H. Kang, ${ }^{37}$ G. Karyan, ${ }^{8}$ H. Kichimi, ${ }^{15}$ B. H. Kim, ${ }^{72}$ C. H. Kim, ${ }^{13}$ D. Y. Kim, ${ }^{74}$ S. H. Kim,${ }^{13}$ K. Kinoshita, ${ }^{7}$ P. Kodyš, ${ }^{5}$ S. Korpar, ${ }^{46,30}$ R. Kroeger, ${ }^{49}$ P. Krokovny, ${ }^{4,64}$ R. Kulasiri, ${ }^{32}$ A. Kuzmin, ${ }^{4,64}$ Y.-J. Kwon, ${ }^{89}$ K. Lalwani, ${ }^{45}$ J. S. Lange ${ }^{10}{ }^{1}$ I. S. Lee, ${ }^{13}$ S. C. Lee, ${ }^{37}$ P. Lewis, ${ }^{14}$ C. H. Li ${ }^{41}$ L. K. Li, ${ }^{24}$ Y. B. Li ${ }^{68}$ L. Li Gioi, ${ }^{47}$ J. Libby, ${ }^{22}$ K. Lieret, ${ }^{43}$ D. Liventsev, ${ }^{86,15}$ C. MacQueen, ${ }^{48}$ M. Masuda, ${ }^{82}$ T. Matsuda, ${ }^{50}$ D. Matvienko, ${ }^{4,64,40}$ M. Merola, ${ }^{27,55}$ K. Miyabayashi, ${ }^{56}$ H. Miyata, ${ }^{62}$ R. Mizuk, ${ }^{40,52}$ R. Mussa, ${ }^{28}$ K. J. Nath ${ }^{20}$ M. Nayak,${ }^{87,15}$ M. Niiyama, ${ }^{36}$ N. K. Nisar, ${ }^{69}$ S. Nishida, ${ }^{15,11}$ K. Nishimura, ${ }^{14}$ S. Ogawa, ${ }^{81}$ H. Ono, ${ }^{61,62}$ Y. Onuki ${ }^{83}$ P. Oskin ${ }^{40}$ P. Pakhlov, ${ }^{40,51}$ G. Pakhlova, ${ }^{40,52}$ T. Pang, ${ }^{69}$ S. Pardi, ${ }^{27}$ H. Park, ${ }^{37}$ S.-H. Park, ${ }^{89}$ S. Patra, ${ }^{19}$ S. Paul, ${ }^{80}$ T. K. Pedlar, ${ }^{44}$ R. Pestotnik, ${ }^{30}$ L. E. Piilonen, ${ }^{86}$ V. Popov, ${ }^{40,52}$ E. Prencipe,${ }^{17}$ M. T. Prim, ${ }^{31}$ M. Röhrken, ${ }^{8}$ A. Rostomyan, ${ }^{8}$ N. Rout ${ }^{22}$ G. Russo,${ }^{55}$ D. Sahoo, ${ }^{79}$ Y. Sakai, ${ }^{15,11}$ S. Sandilya, ${ }^{7}$ L. Santelj, ${ }^{15}$ V. Savinov, ${ }^{69}$ O. Schneider, ${ }^{39}$ G. Schnell, ${ }^{1,18}$ C. Schwanda, ${ }^{25}$ Y. Seino, ${ }^{62}$ K. Senyo ${ }^{88}$ M. E. Sevior, ${ }^{48}$ J.-G. Shiu, ${ }^{59}$ B. Shwartz, ${ }^{4,64}$ A. Sokolov, ${ }^{26}$ E. Solovieva, ${ }^{40}$ S. Stanič ${ }^{63}$ M. Starič ${ }^{30}$ Z. S. Stottler, ${ }^{86}$ T. Sumiyoshi, ${ }^{85}$ W. Sutcliffe, ${ }^{31}$ M. Takizawa ${ }^{73,16,70}$ K. Tanida, ${ }^{29}$ F. Tenchini, ${ }^{8}$ K. Trabelsi, ${ }^{38}$ M. Uchida, ${ }^{84}$ T. Uglov, ${ }^{40,52}$ Y. Unno, ${ }^{13}$ S. Uno, ${ }^{15,11}$ Y. Usov,${ }^{4,64}$ R. Van Tonder, ${ }^{31}$ G. Varner, ${ }^{14}$

A. Vinokurova, ${ }^{4,64}$ V. Vorobyev, ${ }^{4,64,40}$ C. H. Wang, ${ }^{58}$ M.-Z. Wang, ${ }^{59}$ M. Watanabe, ${ }^{62}$ E. Won, ${ }^{35}$ S. B. Yang, ${ }^{35}$ H. Ye, ${ }^{8}$ J. H. Yin, ${ }^{24}$ Z. P. Zhang, ${ }^{71}$ V. Zhilich, ${ }^{4,64}$ V. Zhukova, ${ }^{40}$ and V. Zhulanov ${ }^{4,64}$

(Belle Collaboration)

\author{
${ }^{1}$ University of the Basque Country UPV/EHU, 48080 Bilbao \\ ${ }^{2}$ Beihang University, Beijing 100191 \\ ${ }^{3}$ Brookhaven National Laboratory, Upton, New York 11973 \\ ${ }^{4}$ Budker Institute of Nuclear Physics SB RAS, Novosibirsk 630090 \\ ${ }^{5}$ Faculty of Mathematics and Physics, Charles University, 12116 Prague \\ ${ }^{6}$ Chonnam National University, Gwangju 61186 \\ ${ }^{7}$ University of Cincinnati, Cincinnati, Ohio 45221 \\ ${ }^{8}$ Deutsches Elektronen-Synchrotron, 22607 Hamburg \\ ${ }^{9}$ Key Laboratory of Nuclear Physics and Ion-beam Application (MOE) and Institute of Modern Physics, \\ Fudan University, Shanghai 200443 \\ ${ }^{10}$ Justus-Liebig-Universität Gießen, 35392 Gießen \\ ${ }^{11}$ SOKENDAI (The Graduate University for Advanced Studies), Hayama 240-0193 \\ ${ }^{12}$ Gyeongsang National University, Jinju 52828 \\ ${ }^{13}$ Department of Physics and Institute of Natural Sciences, Hanyang University, Seoul 04763 \\ ${ }^{14}$ University of Hawaii, Honolulu, Hawaii 96822 \\ ${ }^{15}$ High Energy Accelerator Research Organization (KEK), Tsukuba 305-0801 \\ ${ }^{16}$ J-PARC Branch, KEK Theory Center, High Energy Accelerator Research Organization (KEK), \\ Tsukuba 305-0801 \\ ${ }^{17}$ Forschungszentrum Jülich, 52425 Jülich \\ ${ }^{18}$ IKERBASQUE, Basque Foundation for Science, 48013 Bilbao \\ ${ }^{19}$ Indian Institute of Science Education and Research Mohali, SAS Nagar, 140306 \\ ${ }^{20}$ Indian Institute of Technology Guwahati, Assam 781039 \\ ${ }^{21}$ Indian Institute of Technology Hyderabad, Telangana 502285 \\ ${ }^{22}$ Indian Institute of Technology Madras, Chennai 600036 \\ ${ }^{23}$ Indiana University, Bloomington, Indiana 47408 \\ ${ }^{24}$ Institute of High Energy Physics, Chinese Academy of Sciences, Beijing 100049 \\ ${ }^{25}$ Institute of High Energy Physics, Vienna 1050 \\ ${ }^{26}$ Institute for High Energy Physics, Protvino 142281
}


${ }^{27}$ National Institute for Nuclear Physics-Sezione di Napoli, 80126 Napoli

${ }^{28}$ National Institute for Nuclear Physics-Sezione di Torino, 10125 Torino

${ }^{29}$ Advanced Science Research Center, Japan Atomic Energy Agency, Naka 319-1195

${ }^{30}$ J. Stefan Institute, 1000 Ljubljana

${ }^{31}$ Institut für Experimentelle Teilchenphysik, Karlsruher Institut für Technologie, 76131 Karlsruhe

${ }^{32}$ Kennesaw State University, Kennesaw, Georgia 30144

${ }^{33}$ King Abdulaziz City for Science and Technology, Riyadh 11442

${ }^{34}$ Korea Institute of Science and Technology Information, Daejeon 34141

${ }^{35}$ Korea University, Seoul 02841

${ }^{36}$ Kyoto University, Kyoto 606-8502

${ }^{37}$ Kyungpook National University, Daegu 41566

${ }^{38}$ Laboratory of the Linear Accelerator, Univ. Paris-Sud, CNRS/IN2P3, Université Paris-Saclay, Orsay 91898

${ }^{39}$ École Polytechnique Fédérale de Lausanne (EPFL), Lausanne 1015

${ }^{40}$ P.N. Lebedev Physical Institute of the Russian Academy of Sciences, Moscow 119991

${ }^{41}$ Liaoning Normal University, Dalian 116029

${ }^{42}$ Faculty of Mathematics and Physics, University of Ljubljana, 1000 Ljubljana

${ }^{43}$ Ludwig Maximilians University, 80539 Munich

${ }^{44}$ Luther College, Decorah, Iowa 52101

${ }^{45}$ Malaviya National Institute of Technology Jaipur, Jaipur 302017

${ }^{46}$ University of Maribor, 2000 Maribor

${ }^{47}$ Max-Planck-Institut für Physik, 80805 München

${ }^{48}$ School of Physics, University of Melbourne, Victoria 3010

${ }^{49}$ University of Mississippi, University, Mississippi 38677

${ }^{50}$ University of Miyazaki, Miyazaki 889-2192

${ }^{51}$ Moscow Physical Engineering Institute, Moscow 115409

${ }^{52}$ Moscow Institute of Physics and Technology, Moscow Region 141700

${ }^{53}$ Graduate School of Science, Nagoya University, Nagoya 464-8602

${ }^{54}$ Kobayashi-Maskawa Institute, Nagoya University, Nagoya 464-8602

${ }^{55}$ Università di Napoli Federico II, 80055 Napoli

${ }^{56}$ Nara Women's University, Nara 630-8506

${ }^{57}$ National Central University, Chung-li 32054

${ }^{58}$ National United University, Miao Li 36003

${ }^{59}$ Department of Physics, National Taiwan University, Taipei 10617

${ }^{60} \mathrm{H}$. Niewodniczanski Institute of Nuclear Physics, Krakow 31-342

${ }^{61}$ Nippon Dental University, Niigata 951-8580

${ }^{62}$ Niigata University, Niigata 950-2181

${ }^{63}$ University of Nova Gorica, 5000 Nova Gorica

${ }^{64}$ Novosibirsk State University, Novosibirsk 630090

${ }^{65}$ Osaka City University, Osaka 558-8585

${ }^{66}$ Pacific Northwest National Laboratory, Richland, Washington 99352

${ }^{67}$ Panjab University, Chandigarh 160014

${ }^{68}$ Peking University, Beijing 100871

${ }^{69}$ University of Pittsburgh, Pittsburgh, Pennsylvania 15260

${ }^{70}$ Theoretical Research Division, Nishina Center, RIKEN, Saitama 351-0198

${ }^{71}$ University of Science and Technology of China, Hefei 230026

${ }^{72}$ Seoul National University, Seoul 08826

${ }^{73}$ Showa Pharmaceutical University, Tokyo 194-8543

${ }^{74}$ Soongsil University, Seoul 06978

${ }^{75}$ University of South Carolina, Columbia, South Carolina 29208

${ }^{76}$ Sungkyunkwan University, Suwon 16419

${ }^{77}$ School of Physics, University of Sydney, New South Wales 2006

${ }^{78}$ Department of Physics, Faculty of Science, University of Tabuk, Tabuk 71451

${ }^{79}$ Tata Institute of Fundamental Research, Mumbai 400005

${ }^{80}$ Department of Physics, Technische Universität München, 85748 Garching

${ }^{81}$ Toho University, Funabashi 274-8510

${ }^{82}$ Earthquake Research Institute, University of Tokyo, Tokyo 113-0032

${ }^{83}$ Department of Physics, University of Tokyo, Tokyo 113-0033

${ }^{84}$ Tokyo Institute of Technology, Tokyo 152-8550

${ }^{85}$ Tokyo Metropolitan University, Tokyo 192-0397 


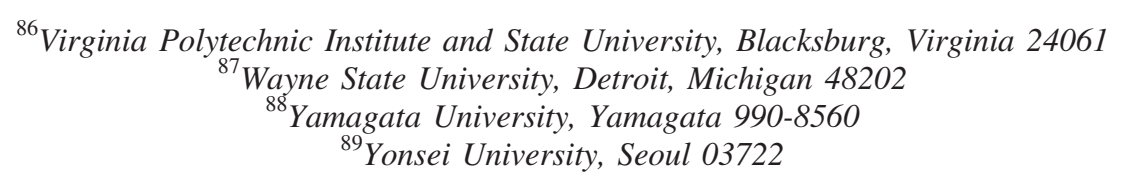

(Received 2 November 2019; revised manuscript received 26 November 2019; published 31 December 2019)

\begin{abstract}
Using a data sample of $921.9 \mathrm{fb}^{-1}$ collected with the Belle detector, we study the process of $e^{+} e^{-} \rightarrow$ $D_{s}^{+} D_{s 1}(2536)^{-}+$c.c. via initial-state radiation. We report the first observation of a vector charmoniumlike state decaying to $D_{s}^{+} D_{s 1}(2536)^{-}+$c.c. with a significance of $5.9 \sigma$, including systematic uncertainties. The measured mass and width are $\left(4625.9_{-6.0}^{+6.2}\right.$ (stat) \pm 0.4 (syst) $) \mathrm{MeV} / c^{2}$ and $\left(49.8_{-11.5}^{+13.9}\right.$ (stat $) \pm 4.0$ (syst) $) \mathrm{MeV}$, respectively. The product of the $e^{+} e^{-} \rightarrow D_{s}^{+} D_{s 1}(2536)^{-}+$c.c. cross section and the branching fraction of $D_{s 1}(2536)^{-} \rightarrow \bar{D}^{* 0} K^{-}$is measured from the $D_{s} \bar{D}_{s 1}(2536)$ threshold to $5.59 \mathrm{GeV}$.
\end{abstract}

DOI: $10.1103 /$ PhysRevD.100.111103

In the past decade, measurements of the exclusive cross sections for $e^{+} e^{-}$annihilation into charmed or charmedstrange meson pairs above the open-charm threshold have attracted much attention [1-10]. These open-charm final states are dominantly produced from the Okubo-ZweigIizuka (OZI)-allowed strong decays of excited vector charmonium states ( $\psi$ states). A comprehensive study of the exclusive $e^{+} e^{-}$cross sections to various open-charm final states could help one to understand the couplings of these $\psi$ states, and extract their resonant parameters.

Many additional $Y$ states with $J^{P C}=1^{--}$with masses above the open-charm threshold have been discovered in the last 14 years [11-19]. It has been noticed that the $Y$ states above the open-charm threshold do not appear explicitly as peaks either in the total hadronic cross section or in the exclusive $e^{+} e^{-}$cross sections to open-charm final states [20] [the only vector charmoniumlike states which reveal themselves as peaks at threshold are the $Y(4630)$ and $Y(4220)$ observed in the $\Lambda_{c}^{+} \bar{\Lambda}_{c}^{-}$and $\pi^{+} D^{0} D^{*-}$ final states, respectively [21,22]]. In $e^{+} e^{-} \rightarrow Y \rightarrow \pi^{+} \pi^{-} J / \psi$ and $\pi^{+} \pi^{-} \psi(2 S) \quad[Y=Y(4260), Y(4660)]$ processes, events in $\pi^{+} \pi^{-}$mass spectra tend to accumulate at the $f_{0}(980)$ nominal mass, which has an $s \bar{s}$ component. Thus, it is natural to search for $Y$ states with a $(c \bar{s})(\bar{c} s)$ quark component. As mentioned in Ref. [23], bound states of $D_{s} \bar{D}_{s}$ mesons, e.g., $D_{s} \bar{D}_{s 1}(2536)$, can appear as a result of $f_{0}(980)$ exchange. Unfortunately, open-charmed-strange production associated with these $Y$ states has not yet been observed.

In this Letter, we perform a measurement of the exclusive cross section for $e^{+} e^{-} \rightarrow D_{s}^{+} D_{s 1}(2536)^{-}\left(D_{s 1}(2536)^{-} \rightarrow\right.$ $\bar{D}^{* 0} K^{-} / D^{*-} K_{S}^{0}$ ) as a function of center-of-mass (C.M.) energy from the $D_{s}^{+} D_{s 1}(2536)^{-}$mass threshold to $5.59 \mathrm{GeV}$

Published by the American Physical Society under the terms of the Creative Commons Attribution 4.0 International license. Further distribution of this work must maintain attribution to the author(s) and the published article's title, journal citation, and DOI. Funded by SCOAP ${ }^{3}$. via initial-state radiation (ISR) [24]. In this process, a charmoniumlike state decaying to $D_{s}^{+} D_{s 1}(2536)^{-}$is observed for the first time. The data used in this analysis correspond to $921.9 \mathrm{fb}^{-1}$ of integrated luminosity at C.M. energies of $10.52,10.58$, and $10.867 \mathrm{GeV}$ collected by the Belle detector [25] at the KEKB asymmetric-energy $e^{+} e^{-}$ collider $[26,27]$.

We use PHOKHARA [28] to generate signal Monte Carlo (MC) events, determine the detector efficiency, and optimize selection criteria for signal events. Generic MC samples of $\Upsilon(4 S) \rightarrow B^{+} B^{-} / B^{0} \bar{B}^{0}, \Upsilon(5 S) \rightarrow B_{s}^{(*)} \bar{B}_{s}^{(*)}$, and $e^{+} e^{-} \rightarrow$ $q \bar{q}(q=u, d, s, c)$ at $\sqrt{s}=10.52,10.58$, and $10.867 \mathrm{GeV}$ with four times the luminosity of data are used to study possible backgrounds.

We fully reconstruct the ISR photon $\gamma_{\mathrm{ISR}}, D_{s}^{+}$, and $K^{-} / K_{S}^{0}$, but do not reconstruct the $\bar{D}^{* 0} / D^{*-}$. Since the $\bar{D}^{* 0} / D^{*-}$ decays are not reconstructed, the detection efficiency for the $e^{+} e^{-} \rightarrow D_{s}^{+} D_{s 1}(2536)^{-}\left(\rightarrow \bar{D}^{* 0} K^{-} / D^{*-} K_{S}^{0}\right)$ process is greatly improved. For the measurement of the $e^{+} e^{-} \rightarrow D_{s}^{+} D_{s 1}(2536)^{-}$cross section, we determine the invariant mass spectrum of $D_{s}^{+} D_{s 1}(2536)^{-}$ $\left(M\left(D_{s}^{+} D_{s 1}(2536)^{-}\right)\right)$, which is equivalent to the mass recoiling against $\gamma_{\text {ISR }}\left(M_{\text {rec }}\left(\gamma_{\text {ISR }}\right)\right)$. Here, $M_{\text {rec }}\left(\gamma_{\text {ISR }}\right)$ is calculated using $M_{\mathrm{rec}}\left(\gamma_{\mathrm{ISR}}\right)=\sqrt{\left(P_{\mathrm{C} . \mathrm{M} .}-P_{\gamma_{\mathrm{ISR}}}\right)^{2}}$, where $P_{\mathrm{C} . \mathrm{M} \text {. }}$. and $P_{\gamma_{\mathrm{ISR}}}$ are the four-momenta of the initial $e^{+} e^{-}$ system and the ISR photon, respectively. However, the energy resolution of $\gamma_{\mathrm{ISR}}$ is very poor due to its high energy. We constrain the recoil mass of the $\gamma_{\mathrm{ISR}} D_{s}^{+} K^{-} / \gamma_{\mathrm{ISR}} D_{s}^{+} K_{S}^{0}$ to the nominal mass of the $\bar{D}^{* 0} / D^{*-}$ meson [29] to improve the resolution for the ISR photon for events within the $\bar{D}^{* 0} / D^{*-}$ signal region. Before applying the mass constraint, the mass resolution of the $M\left(D_{s}^{+} D_{s 1}(2536)^{-}\right)$system is about $180 \mathrm{MeV} / c^{2}$. As a result of the constraint, the mass resolution is significantly improved, to about $5 \mathrm{MeV} / c^{2}$.

The $D_{s}^{+}$candidates are reconstructed using eight decay modes: $\phi \pi^{+}, \bar{K}^{*}(892)^{0} K^{+}, K_{S}^{0} K^{+}, K^{+} K^{-} \pi^{+} \pi^{0}, K_{S}^{0} \pi^{0} K^{+}$, $K^{*}(892)^{+} K_{S}^{0}, \eta \pi^{+}$, and $\eta^{\prime} \pi^{+}$. We use the techniques of Ref. [30] to reconstruct particles such as photons, charged 
pions and kaons, and $K_{S}^{0}$. The $\phi, \bar{K}^{*}(892)^{0}$, and $K^{*}(892)^{+}$ candidates are reconstructed in the $K^{+} K^{-}, K^{-} \pi^{+}$, and $K_{S}^{0} \pi^{+}$decay modes. The invariant masses of the $K_{S}^{0}, \phi$, $\bar{K}^{*}(892)^{0}$, and $K^{*}(892)^{+}$candidates are required to be within $10,10,50$, and $50 \mathrm{MeV} / c^{2}$ of the corresponding nominal masses [29] (>95\% signal events are retained), respectively.

The most energetic ISR photon is required to have energy greater than $3 \mathrm{GeV}$ in the $e^{+} e^{-}$C.M. frame. Pairs of photons are combined to form $\pi^{0}$ candidates. The energies of the photons from $\pi^{0}$ are required to be greater than $50 \mathrm{MeV}$ in the calorimeter barrel and $100 \mathrm{MeV}$ in the calorimeter end caps [31] in the laboratory frame. The $\eta$ candidates are reconstructed via $\gamma \gamma$ and $\pi^{+} \pi^{-} \pi^{0}$ decay modes. Photon candidates from $\eta \rightarrow \gamma \gamma$ are required to have energies greater than $100 \mathrm{MeV}$ in the laboratory frame. The reconstructed $\eta$ candidates are then combined with $\pi^{+} \pi^{-}$pairs to form $\eta^{\prime}$ candidates. The mass windows applied for $\pi^{0}, \eta \rightarrow \gamma \gamma, \eta \rightarrow \pi^{+} \pi^{-} \pi^{0}$, and $\eta^{\prime}$ candidates are $\pm 12, \pm 20, \pm 10$, and $\pm 10 \mathrm{MeV} / c^{2}$, which are within approximately $2.5 \sigma$ of the corresponding meson nominal masses [29]. After applying the mass window requirements, mass-constrained fits are applied to the $\pi^{0}, \eta$, and $\eta^{\prime}$ candidates to improve their momentum resolutions.

Before calculation of the $D_{s}^{+}$candidate mass, a fit to a common vertex is performed for charged tracks in the $D_{s}^{+}$candidate. After the application of the above requirements, $D_{s}^{+}$signals are clearly observed. We define the $D_{s}^{+}$signal region as $\left|M\left(D_{s}^{+}\right)-m_{D_{s}^{+}}\right|<12 \mathrm{MeV} / c^{2}$ $(\sim 2.0 \sigma)$. Here and throughout the text, $m_{i}$ represents the nominal mass of particle $i$ [29]. To improve the momentum resolution of the $D_{s}^{+}$meson candidate, a mass-constrained fit to the $D_{s}^{+}$nominal mass [29] is performed. The $D_{s}^{+}$mass sideband regions are defined as $1912.34<M\left(D_{s}^{+}\right)<1936.34 \mathrm{MeV} / \mathrm{c}^{2}$ and $2000.34<$ $M\left(D_{s}^{+}\right)<2024.34 \mathrm{MeV} / \mathrm{c}^{2}$, which are twice as wide as the signal region. The $D_{s}^{+}$candidates from the sidebands are also constrained to the central mass values in the defined $D_{s}^{+}$ sideband regions. The $D_{s}^{+}$candidate with the smallest $\chi^{2}$ from the $D_{s}^{+}$mass fit is kept. Besides the selected ISR photon and $D_{s}^{+}$, we require at least one additional $K^{-}$or $K_{S}^{0}$ candidate in the event, and retain all the combinations (the fraction of events with multiple candidates is $1.7 \%$ ).

Figure 1(a) shows the sum of the recoil mass spectra against the $\gamma_{\mathrm{ISR}} D_{s}^{+} K^{-}$and $\gamma_{\mathrm{ISR}} D_{s}^{+} K_{S}^{0}$ systems after requiring the events be within the $D_{s 1}(2536)^{-}$signal region (see below) in data. Due to the poor recoil mass resolution, the $\bar{D}^{* 0} / D^{*-}$ signal is very wide. The $\bar{D}^{* 0} / D^{*-}$ signal component is modeled using a Gaussian function convolved with a Novosibirsk function [32] derived from the signal MC samples, while the combinatorial backgrounds are described by a second-order polynomial. The solid curve is the total fit; the $\bar{D}^{* 0} / D^{*-}$ signal yield is $275 \pm 32$. We define an asymmetric requirement of $-200<M_{\mathrm{rec}}\left(\gamma_{\mathrm{ISR}} D_{S}^{+} K^{-} / K_{S}^{0}\right)-$ $m_{\bar{D}^{* 0} / D^{*-}}<400 \mathrm{MeV} / c^{2}$ for the $\bar{D}^{* 0} / D^{*-}$ signal region.
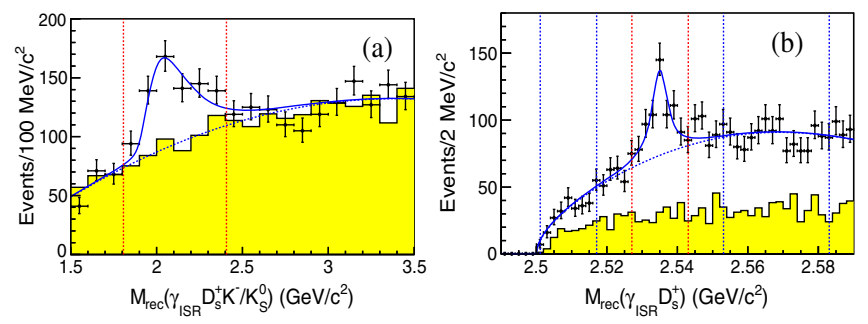

FIG. 1. (a) The recoil mass spectrum against the $\gamma_{\mathrm{ISR}} D_{S}^{+} K^{-} / K_{S}^{0}$ system before applying the $\bar{D}^{* 0} / D^{*-}$ mass constraint. The yellow histogram shows the normalized $D_{s 1}(2536)^{-}$mass sidebands (see below). The red dashed lines show the required $\bar{D}^{* 0} / D^{*-}$ signal region. (b) The recoil mass spectrum against the $\gamma_{\mathrm{ISR}} D_{s}^{+}$system in data. The yellow histogram shows the normalized $D_{s}^{+}$mass sidebands. The red dashed lines show the required $D_{s 1}(2536)^{-}$ signal region, and the blue dashed lines show the $D_{s 1}(2536)^{-}$ mass sidebands.

Hereinafter the $\bar{D}^{* 0} / D^{*-}$ mass constraint is applied for events in the $\bar{D}^{* 0} / D^{*-}$ signal region to improve mass resolution.

The recoil mass spectrum against the $\gamma_{\mathrm{ISR}} D_{s}^{+}$system after requiring the events within $\bar{D}^{* 0} / D^{*-}$ signal region is shown in Fig. 1(b). A clear $D_{s 1}(2536)^{-}$signal is observed. The signal shape is described by a double Gaussian function (all the parameters are fixed to those from a fit to the MC simulated distribution), and a threshold function is used for the backgrounds. The threshold function is $\left(M_{\mathrm{rec}}-x_{\mathrm{thr}}\right)^{\alpha} e^{\left[\beta_{1}\left(M_{\mathrm{rec}}-x_{\mathrm{thr}}\right)+\beta_{2}\left(M_{\mathrm{rec}}-x_{\mathrm{thr}}\right)^{2}\right]}$, where $M_{\mathrm{rec}}$ is the recoil mass of the $\gamma_{\mathrm{ISR}} D_{s}^{+}$; the parameters $\alpha, \beta_{1}$, and $\beta_{2}$ are free; the threshold parameter $x_{\mathrm{thr}}$ is fixed from generic MC simulations. The fit yields $254 \pm 36$ $D_{s 1}(2536)^{-}$signal events as shown in Fig. 1(b) [33]. We define the $D_{s 1}(2536)^{-}$signal region as $\mid M_{\mathrm{rec}}\left(\gamma_{\mathrm{ISR}} D_{s}^{+}\right)-$ $m_{D_{s 1}(2536)^{-}} \mid<8 \mathrm{MeV} / c^{2}(\sim 2.5 \sigma)$, and sideband regions as shown by blue dashed lines, which are three times as wide as the signal region. To estimate the signal significance of the $D_{s 1}(2536)^{-}$, we compute $\sqrt{-2 \ln \left(\mathcal{L}_{0} / \mathcal{L}_{\max }\right)}$ [34], where $\mathcal{L}_{0}$ and $\mathcal{L}_{\max }$ are the maximized likelihoods without and with the $D_{s 1}(2536)^{-}$signal, respectively. The statistical significance of the $D_{s 1}(2536)^{-}$signal is $8 \sigma$.

The $D_{s}^{+} D_{s 1}(2536)^{-}$invariant mass distribution is shown in Fig. 2(a). There is a significant peak around $4626 \mathrm{MeV} / c^{2}$, while no structure is seen in the normalized $D_{s 1}(2536)^{-}$mass sidebands shown as the yellow histogram. In addition, no peaking background is found in the $D_{s}^{+} D_{s 1}(2536)^{-}$mass distribution from generic MC samples. We therefore interpret the peak in the data as evidence for an exotic charmoniumlike state [35] decaying into $D_{s}^{+} D_{s 1}(2536)^{-}$, called $Y(4626)$ hereafter.

One possible background, which is not included in the $D_{s 1}(2536)^{-}$mass sidebands, is from $e^{+} e^{-} \rightarrow$ $D_{s}^{*+}\left(\rightarrow D_{s}^{+} \gamma\right) D_{s 1}(2536)^{-}$, where the photon from the $D_{s}^{*+}$ remains undetected. To estimate such a background contribution, we measure this process with the data 

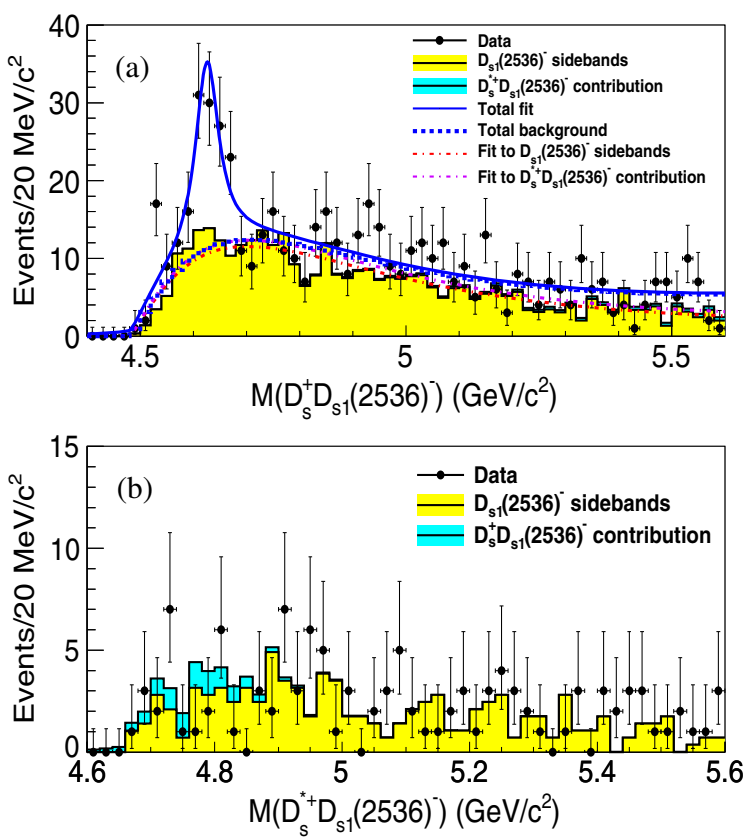

FIG. 2. (a) The $D_{s}^{+} D_{s 1}(2536)^{-}$invariant mass spectrum for $e^{+} e^{-} \rightarrow D_{s}^{+} D_{s 1}(2536)^{-}$. (b) The $D_{s}^{*+} D_{s 1}(2536)^{-}$invariant mass spectrum for $e^{+} e^{-} \rightarrow D_{s}^{*+} D_{s 1}(2536)^{-}$. All the components including those from the fit to the $D_{s}^{+} D_{s 1}(2536)^{-}$invariant mass spectrum are indicated in the labels and described in the text. Note that the cyan shaded histograms in the top/bottom show the $D_{s}^{+} D_{s 1}(2536)^{-} / D_{s}^{*+} D_{s 1}(2536)^{-}$invariant mass spectrum from $D_{s}^{*+} D_{s 1}(2536)^{-} / D_{s}^{+} D_{s 1}(2536)^{-}$background contribution after applying the requirements to reconstruct $e^{+} e^{-} \rightarrow$ $D_{s}^{+} D_{s 1}(2536)^{-} / e^{+} e^{-} \rightarrow D_{s}^{*+} D_{s 1}(2536)^{-}$.

following the same procedure as used for the signal process. We require an extra photon with $E_{\gamma}>50 \mathrm{MeV}$ in the barrel or $E_{\gamma}>100 \mathrm{MeV}$ in the end caps to combine with the $D_{s}^{+}$to form the $D_{s}^{*+}$ candidate. The mass and vertex fits are applied to the $D_{s}^{*+}$ candidates to improve their momentum resolution. In events with multiple candidates, the best candidate is chosen using the lowest $\chi^{2}$ value from the mass-constrained fit. The same $\bar{D}^{* 0} / D^{*-}$ signal region requirement on $M_{\mathrm{rec}}\left(\gamma_{\mathrm{ISR}} D_{s}^{*+} K^{-} / K_{S}^{0}\right)$ and the $\bar{D}^{* 0} / D^{*-}$ mass constraint are applied as before in $e^{+} e^{-} \rightarrow D_{s}^{+} D_{s 1}(2536)^{-}$. In the recoil mass spectrum of the $\gamma_{\mathrm{ISR}} D_{s}^{*+}$ an excess of $28 \pm 13 D_{s 1}(2536)^{-}$signal events with a statistical significance of $2.4 \sigma$ is observed in the $D_{s 1}(2536)^{-}$signal region.

After requiring the $D_{s}^{+} K^{-} / K_{S}^{0}$ mass to be within the $D_{s 1}(2536)^{-}$signal region, the $D_{s}^{*+} D_{s 1}(2536)^{-}$invariant mass distribution is shown in Fig. 2(b). Note that the process $e^{+} e^{-} \rightarrow D_{s}^{+} D_{s 1}(2536)^{-}$is a source of backgrounds for the $e^{+} e^{-} \rightarrow D_{s}^{*+} D_{s 1}(2536)^{-}$when the $D_{s}^{+}$ candidates are combined with low energy photons to form $D_{s}^{*+}$ candidates. From Fig. 2(b), no obvious structure is observed. The normalized contribution from $e^{+} e^{-} \rightarrow D_{s}^{*+} D_{s 1}(2536)^{-}$to $e^{+} e^{-} \rightarrow D_{s}^{+} D_{s 1}(2536)^{-}$ is the cyan shaded histogram which is shown in
Fig. 2(a), and which is normalized to correspond to $N_{D_{s}^{*+} D_{s 1}(2536)^{-}}^{\text {obs }} \varepsilon_{D_{s}^{+} D_{s 1}(2536)^{-}} / \varepsilon_{D_{s}^{*+} D_{s 1}(2536)^{-}}$events. Here $N_{D_{s}^{*+} D_{s 1}(2536)^{-}}^{\text {obs }}$ is the yield of $e^{+} e^{-} \rightarrow D_{s}^{*+} D_{s 1}(2536)^{-}$ signal events in each $M\left(D_{s}^{*+} D_{s 1}(2536)^{-}\right)$bin in data after subtracting the normalized $D_{s 1}(2536)^{-}$sidebands and the $e^{+} e^{-} \rightarrow D_{s}^{+} D_{s 1}(2536)^{-}$background contribution, and $\varepsilon_{D_{s}^{+} D_{s 1}(2536)^{-}}$and $\varepsilon_{D_{s}^{*+} D_{s 1}(2536)^{-}}$are the reconstruction efficiencies for $e^{+} e^{-} \rightarrow D_{s}^{+} D_{s 1}(2536)^{-}$and $e^{+} e^{-} \rightarrow$ $D_{s}^{*+} D_{s 1}(2536)^{-}$, respectively, where the ratio of efficiencies is $(1.00 \pm 0.02)$. The yield of $D_{s}^{*+} D_{s 1}(2536)^{-}$after background subtraction for the entire region in Fig. 2(b) is $(11.6 \pm 3.6)$. A similar method is applied to estimate the background contribution from $e^{+} e^{-} \rightarrow D_{s}^{+} D_{s 1}(2536)^{-}$ to $e^{+} e^{-} \rightarrow D_{s}^{*+} D_{s 1}(2536)^{-}$.

We perform an unbinned likelihood fit simultaneously to the $M\left(D_{s}^{+} D_{s 1}(2536)^{-}\right)$distributions of all selected $D_{s 1}(2536)^{-}$signal candidates, the normalized $D_{s 1}(2536)^{-}$ mass sidebands, and the $e^{+} e^{-} \rightarrow D_{s}^{*+} D_{s 1}(2536)^{-}$contribution. The yields in the normalized $D_{s 1}(2536)^{-}$mass sidebands and the $e^{+} e^{-} \rightarrow D_{s}^{*+} D_{s 1}(2536)^{-}$contribution are fixed in the fit. The following components are included in the fit to the $M\left(D_{s}^{+} D_{s 1}(2536)^{-}\right)$distribution: a resonance signal, a nonresonant contribution, the $D_{s 1}(2536)^{-}$mass sidebands, and an $e^{+} e^{-} \rightarrow D_{s}^{*+} D_{s 1}(2536)^{-}$contribution. A Breit-Wigner (BW) function convolved with a Gaussian function (with its width fixed at $5.0 \mathrm{MeV} / \mathrm{c}^{2}$ according to the MC simulation), multiplied by an efficiency function that has a linear dependence on $M\left(D_{s}^{+} D_{s 1}(2536)^{-}\right)$ and the differential ISR effective luminosity [36], is taken as the signal shape. Here the BW formula used has the form [37]

$$
B W(\sqrt{s})=\frac{\sqrt{12 \pi \Gamma_{e e} \mathcal{B}_{f} \Gamma}}{s-M^{2}+i M \Gamma} \sqrt{\frac{\Phi_{2}(\sqrt{s})}{\Phi_{2}(M)}},
$$

where $M$ is the mass of the resonance, $\Gamma$ and $\Gamma_{e e}$ are the total width and partial width to $e^{+} e^{-}, \mathcal{B}_{f}=\mathcal{B}(Y(4626) \rightarrow$ $\left.D_{s}^{+} D_{s 1}(2536)^{-}\right) \times \mathcal{B}\left(D_{s 1}(2536)^{-} \rightarrow \bar{D}^{* 0} K^{-}\right)$is the product branching fraction of the $Y(4626)$ into the final state, and $\Phi_{2}$ is the two-body decay phase space factor that increases smoothly from the mass threshold with $\sqrt{s}$, respectively. A two-body phase space form is also taken into account for the nonresonant contribution. The $D_{s 1}(2536)^{-}$mass sidebands and the $e^{+} e^{-} \rightarrow$ $D_{s}^{*+} D_{s 1}(2536)^{-}$contribution are parametrized with threshold functions.

The fit results are shown in Fig. 2(a), where the solid blue curve is the best fit, the blue dotted curve is the sum of the backgrounds, the red dot-dashed curve is the fitted result to the normalized $D_{s 1}(2536)^{-}$mass sidebands, and the violet dot-dashed curve is for the $e^{+} e^{-} \rightarrow$ $D_{s}^{*+} D_{s 1}(2536)^{-}$contribution. The yield of the $Y(4626)$ signal is $89_{-16}^{+17}$. The statistical significance of the $Y(4626)$ 
signal is $6.5 \sigma$, calculated from the difference of the logarithmic likelihoods [34], $-2 \ln \left(\mathcal{L}_{0} / \mathcal{L}_{\text {max }}\right)=50.4$, where $\mathcal{L}_{0}$ and $\mathcal{L}_{\max }$ are the maximized likelihoods without and with a signal component, respectively, taking into account the difference in the number of degrees of freedom $(\Delta \mathrm{ndf}=3)$. The parametrization of the nonresonant contribution is the dominant systematic uncertainty for the estimate of the signal significance. Changing the twobody phase space form to a threshold function parametrized by $\sqrt{M-x_{\text {thr }}}$ or a two-body phase space form plus a threshold function, the $Y(4626)$ signal significance is reduced to $5.9 \sigma$. We take this value as the signal significance with systematic uncertainties included. The fitted mass and width for the $Y(4626)$ are $\left(4625.9_{-6.0}^{+6.2}\right.$ (stat $) \pm$ 0.4 (syst) $) \mathrm{MeV} / c^{2}$ and $\left(49.8_{-11.5}^{+13.9}\right.$ (stat) \pm 4.0 (syst) $) \mathrm{MeV}$, respectively. The value of $\Gamma_{e e} \times \mathcal{B}(Y(4626) \rightarrow$ $\left.D_{s}^{+} D_{s 1}(2536)^{-}\right) \times \mathcal{B}\left(D_{s 1}(2536)^{-} \rightarrow \bar{D}^{* 0} K^{-}\right)$is obtained to be $\left(14.3_{-2.6}^{+2.8}\right.$ (stat) \pm 1.5 (syst) $) \mathrm{eV}$. The systematic uncertainties are discussed below.

The $e^{+} e^{-} \rightarrow D_{s}^{+} D_{s 1}(2536)^{-}$cross section is extracted from the recoil mass spectrum against the $\gamma_{\mathrm{ISR}} D_{s}^{+}$ system. The product of the $e^{+} e^{-} \rightarrow D_{s}^{+} D_{s 1}(2536)^{-}$dressed cross section $(\sigma)$ [38] and the decay branching fraction $\mathcal{B}\left(D_{s 1}(2536)^{-} \rightarrow \bar{D}^{* 0} K^{-}\right)$for each $D_{s}^{+} D_{s 1}(2536)^{-}$mass bin from threshold to $5.59 \mathrm{GeV} / c^{2}$ in steps of $20 \mathrm{MeV} / c^{2}$ is computed as

$$
\begin{gathered}
\sigma\left(e^{+} e^{-} \rightarrow D_{s}^{+} D_{s 1}(2536)^{-}\right) \mathcal{B}\left(D_{s 1}(2536)^{-} \rightarrow \bar{D}^{* 0} K^{-}\right) \\
=\frac{N_{\mathrm{fit}}^{D_{s 1}(2536)^{-}}}{d \mathcal{L} \times\left[\Sigma_{i}\left(\varepsilon_{i}^{\bar{D}^{* 0} K^{-}} \times \mathcal{B}_{i}\right)+R_{\bar{D}^{* 0} K^{-}}^{D^{*-} K^{0}} \times \Sigma_{i}\left(\varepsilon_{i}^{D^{*-} K_{S}^{0}} \times \mathcal{B}_{i}\right)\right]},
\end{gathered}
$$

where $N_{\text {fit }}^{D_{s 1}(2536)^{-}}$is the yield of fitted $D_{s 1}(2536)^{-}$signal events after subtracting the $e^{+} e^{-} \rightarrow D_{s}^{*+} D_{s 1}(2536)^{-}$ background contribution in data, $d \mathcal{L}$ is the effective luminosity [36], $\quad \Sigma_{i}\left(\varepsilon_{i}^{\bar{D}^{* 0} K^{-}} \times \mathcal{B}_{i}\right)$ and $\quad \Sigma_{i}\left(\varepsilon_{i}^{D^{*-} K_{S}^{0}} \times \mathcal{B}_{i}\right)$ are the sums of the product of the reconstruction efficiency and branching fraction for each $D_{s}^{+}$decay mode (i) in $D_{s 1}(2536)^{-} \rightarrow \bar{D}^{* 0} K^{-}$and $D_{s 1}(2536)^{-} \rightarrow D^{*-} K_{S}^{0}$, and $R_{\bar{D}^{* 0} K^{-}}^{D^{*-}}=\mathcal{B}\left(D_{s 1}(2536)^{-} \rightarrow D^{*-} K_{S}^{0}\right) / \mathcal{B}\left(D_{s 1}(2536)^{-} \rightarrow\right.$ $\left.\bar{D}^{* 0} K^{-}\right)=0.425 \pm 0.06$ taken from Ref. [29]. The values used to calculate $\sigma\left(e^{+} e^{-} \rightarrow D_{s}^{+} D_{s 1}(2536)^{-}\right) \times$ $\mathcal{B}\left(D_{s 1}(2536)^{-} \rightarrow \bar{D}^{* 0} K^{-}\right)$are summarized in Supplemental Material [33]. In the fit to the recoil mass spectrum of $\gamma_{\mathrm{ISR}} D_{s}^{+}$combinations in each $D_{s}^{+} D_{s 1}(2536)^{-}$ mass bin, the $D_{s 1}(2536)^{-}$signal shape is fixed to that from the overall fit, as shown by the blue solid curve in Fig. 1, and a threshold function is used for the backgrounds. The resulting $\sigma\left(e^{+} e^{-} \rightarrow D_{s}^{+} D_{s 1}(2536)^{-}\right) \times \mathcal{B}\left(D_{s 1}(2536)^{-} \rightarrow\right.$ $\left.\bar{D}^{* 0} K^{-}\right)$value as a function of $M\left(D_{s}^{+} D_{s 1}(2536)^{-}\right)$is shown in Fig. 3 with the statistical and systematic uncertainties discussed below summed in quadrature.

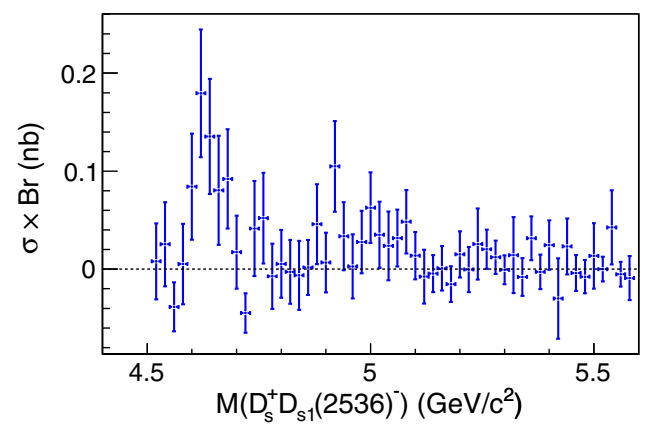

FIG. 3. The product of the $e^{+} e^{-} \rightarrow D_{s}^{+} D_{s 1}(2536)^{-}$cross section and branching fraction $\mathcal{B}\left(D_{s 1}(2536)^{-} \rightarrow \bar{D}^{* 0} K^{-}\right)$as a function of $M\left(D_{s}^{+} D_{s 1}(2536)^{-}\right)$; here the statistical and systematic uncertainties are summed in quadrature. Here, the correlated systematic uncertainties from the detection efficiency, branching fraction, and luminosity are not included.

The sources of systematic uncertainties for the cross section measurement include detection-efficiency-related uncertainties, branching fractions of the intermediate states, fit uncertainty, resonance parameters, the $\mathrm{MC}$ event generator, $e^{+} e^{-} \rightarrow D_{s}^{*+} D_{s 1}(2536)^{-}$background contribution, mass resolution as well as the integrated luminosity. The detection-efficiency-related uncertainties include those for tracking efficiency $(0.35 \% /$ track $)$, particle identification efficiency $\left(1.1 \% /\right.$ kaon and $0.9 \% /$ pion), $K_{S}^{0}$ selection efficiency $(1.4 \%)$ [40], $\pi^{0}$ reconstruction efficiency $\left(2.25 \% / \pi^{0}\right)$ and photon reconstruction efficiency (2.0\%/photon). The above individual uncertainties from different $D_{s}^{+}$decay channels are added linearly, weighted by the product of the detection efficiency and $D_{s}^{+}$partial decay width. These uncertainties are summed in quadrature to obtain the final uncertainty related to the reconstruction efficiency. Uncertainties for $D_{s}^{+}$decay branching fractions and $R_{\bar{D}^{* 0} K_{s}^{-}}^{D^{*} K^{0}}$ are taken from Ref. [29]; the final uncertainties on the $D_{s}^{+}$ partial decay widths are summed in quadrature over the eight $D_{s}^{+}$decay modes weighted by the product of the efficiency and the $D_{s}^{+}$partial decay width. Systematic uncertainties associated with the fitting procedure are estimated by changing the order of the background polynomial and the range of the fit. The deviations from nominal fit results are taken as systematic uncertainties. Changing the values of mass and width of $D_{s 1}(2536)^{-}$by $1 \sigma$ [29] in each $M\left(D_{s}^{+} D_{s 1}(2536)^{-}\right)$bin has no effect on the fits. Thus, the uncertainty from the resonance parameters can be neglected. The PHOKHARA generator calculates the ISR-photon radiator function with $0.1 \%$ accuracy [28]. The uncertainty attributed to the generator can also be neglected.

By fitting the $D_{s 1}(2536)^{-}$mass spectrum in each $M\left(D_{s}^{*+} D_{s 1}(2536)^{-}\right)$bin for $e^{+} e^{-} \rightarrow D_{s}^{*+} D_{s 1}(2536)^{-}$, we find the signal yields are less than 1 . In addition, the $D_{s 1}(2536)^{-}$signal from the $e^{+} e^{-} \rightarrow D_{s}^{*+} D_{s 1}(2536)^{-}$contribution has a much poorer mass resolution according to 
TABLE I. Summary of the absolute systematic uncertainties $\left(\sigma_{\text {sys }}\right)$ on the product of $e^{+} e^{-} \rightarrow D_{s}^{+} D_{s 1}(2536)^{-}$cross section and the decay branching fraction $\mathcal{B}\left(D_{s 1}(2536)^{-} \rightarrow \bar{D}^{* 0} K^{-}\right)$for different $M\left(D_{s}^{+} D_{s 1}(2536)^{-}\right)$bins.

\begin{tabular}{ll}
\hline \hline Source & $\sigma_{\text {sys }}(\mathrm{pb})$ \\
\hline Detection efficiency & $0.0-8.1$ \\
Branching fractions & $0.0-7.4$ \\
Fit uncertainty & $0.5-36.2$ \\
Mass resolution & $0.1-7.7$ \\
Luminosity & $0.0-2.5$ \\
Quadratic sum & $0.6-36.3$ \\
\hline \hline
\end{tabular}

MC simulation. Therefore, the systematic uncertainty associated with the $e^{+} e^{-} \rightarrow D_{s}^{*+} D_{s 1}(2536)^{-}$contribution is neglected. The MC simulation is known to reproduce the resolution of mass peaks within $10 \%$ over a large number of different systems. The systematic uncertainty in the mass resolution is estimated by comparing the yields when the mass resolution is changed by $10 \%$. The total luminosity is determined to $1.4 \%$ precision using wide-angle Bhabha scattering events. All the uncertainties are summarized in Table I. Assuming all the sources are independent, we sum them in quadrature to obtain the total systematic uncertainties.

The following systematic uncertainties on the measured mass and width for the $Y(4626)$, and the $\Gamma_{e e} \times$ $\mathcal{B}\left(Y(4626) \rightarrow D_{s}^{+} D_{s 1}(2536)^{-}\right) \times \mathcal{B}\left(D_{s 1}(2536)^{-} \rightarrow \bar{D}^{* 0} K^{-}\right)$ are considered. The resultant systematic uncertainties attributed to the mass resolution in the width and $\Gamma_{e e} \times$ $\mathcal{B}\left(Y(4626) \rightarrow D_{s}^{+} D_{s 1}(2536)^{-}\right) \times \mathcal{B}\left(D_{s 1}(2536)^{-} \rightarrow \bar{D}^{* 0} K^{-}\right)$ are $0.3 \mathrm{MeV}$ and $0.1 \mathrm{eV}$. By changing the nonresonant background shape to a threshold function or to the sum of a two-body phase space form and a threshold function, the differences of $0.3 \mathrm{MeV} / c^{2}$ and $3.9 \mathrm{MeV}$ in the measured mass and width, and $1.3 \mathrm{eV}$ for the $\Gamma_{e e} \times \mathcal{B}(Y(4626) \rightarrow$ $\left.D_{s}^{+} D_{s 1}(2536)^{-}\right) \times \mathcal{B}\left(D_{s 1}(2536)^{-} \rightarrow \bar{D}^{* 0} K^{-}\right)$, respectively, are taken as systematic uncertainties. The uncertainty in the efficiency correction from detection efficiency, branching fractions of the intermediate states, and integrated luminosity is $4.9 \%$. Changing the efficiency function by $4.9 \%$ gives a $0.1 \mathrm{MeV} / c^{2}$ change on the mass, $0.2 \mathrm{MeV}$ on the width, and $0.7 \mathrm{eV}$ on the product $\Gamma_{e e} \times \mathcal{B}(Y(4626) \rightarrow$ $\left.D_{s}^{+} D_{s 1}(2536)^{-}\right) \times \mathcal{B}\left(D_{s 1}(2536)^{-} \rightarrow \bar{D}^{* 0} K^{-}\right)$. Finally, the total systematic uncertainties on the $Y(4626)$ mass, width, and $\Gamma_{e e} \times \mathcal{B}\left(Y(4626) \rightarrow D_{s}^{+} D_{s 1}(2536)^{-}\right) \times \mathcal{B}\left(D_{s 1}(2536)^{-} \rightarrow\right.$ $\left.\bar{D}^{* 0} K^{-}\right)$are $0.4 \mathrm{MeV} / c^{2}, 4.0 \mathrm{MeV}$, and $1.5 \mathrm{eV}$, respectively.

In summary, the product of the $e^{+} e^{-} \rightarrow D_{s}^{+} D_{s 1}(2536)^{-}$ cross section and the decay branching fraction $\mathcal{B}\left(D_{s 1}(2536)^{-} \rightarrow \bar{D}^{* 0} K^{-}\right)$is measured over the C.M. energy range from the $D_{s}^{+} D_{s 1}(2536)^{-}$mass threshold to $5.59 \mathrm{GeV}$ for the first time. We observe the first vector charmoniumlike state decaying to a charmedantistrange and anticharmed-strange meson pair $D_{s}^{+} D_{s 1}(2536)^{-}$with a signal significance of $5.9 \sigma$ with systematic uncertainties included. The measured mass and width are $\left(4625.9_{-6.0}^{+6.2}\right.$ (stat) \pm 0.4 (syst) $) \mathrm{MeV} / c^{2}$ and $\left(49.8_{-11.5}^{+13.9}\right.$ (stat) \pm 4.0 (syst) $) \mathrm{MeV}$, respectively, which are consistent with the $Y(4660)$ mass of $(4643 \pm 9) \mathrm{MeV} / c^{2}$ and width of $(72 \pm 11) \mathrm{MeV}$ [29] within uncertainties. The $\Gamma_{e e} \times \mathcal{B}\left(Y(4626) \rightarrow D_{s}^{+} D_{s 1}(2536)^{-}\right) \times \mathcal{B}\left(D_{s 1}(2536)^{-} \rightarrow\right.$ $\left.\bar{D}^{* 0} K^{-}\right)$is obtained to be $\left(14.3_{-2.6}^{+2.8}(\right.$ stat $) \pm 1.5$ (syst) $) \mathrm{eV}$.

\section{ACKNOWLEDGEMENT}

We thank the KEKB group for the excellent operation of the accelerator; the KEK cryogenics group for the efficient operation of the solenoid; and the KEK computer group, and the Pacific Northwest National Laboratory (PNNL) Environmental Molecular Sciences Laboratory (EMSL) computing group for strong computing support; and the National Institute of Informatics, and Science Information NETwork 5 (SINET5) for valuable network support. We acknowledge support from the Ministry of Education, Culture, Sports, Science, and Technology (MEXT) of Japan, the Japan Society for the Promotion of Science (JSPS), and the Tau-Lepton Physics Research Center of Nagoya University; the Australian Research Council including Grants No. DP180102629, No. DP170102389, No. DP170102204, No. DP150103061, and No. FT130100303; Austrian Science Fund (FWF); the National Natural Science Foundation of China under Contracts No. 11435013, No. 11475187, No. 11521505, No. 11575017, No. 11675166, No. 11705209, No. 11761141009, No. 11975076; Key Research Program of Frontier Sciences, Chinese Academy of Sciences (CAS), Grant No. QYZDJ-SSW-SLH011; the CAS Center for Excellence in Particle Physics (CCEPP); the Shanghai Pujiang Program under Grant No. 18PJ1401000; the Ministry of Education, Youth and Sports of the Czech Republic under Contract No. LTT17020; the Carl Zeiss Foundation, the Deutsche Forschungsgemeinschaft, the Excellence Cluster Universe, and the VolkswagenStiftung; the Department of Science and Technology of India; the Istituto Nazionale di Fisica Nucleare of Italy; National Research Foundation (NRF) of Korea Grants No. 2016R1-D1A1B-01010135, No. 2016R1-D1A1B-02012900, No. 2018R1-A2B3003643, No. 2018R1-A6A1A-06024970, No. 2018R1D1A1B-07047294, No. 2019K1-A3A7A-09033840, and No. 2019R1-I1A3A-01058933; Radiation Science Research Institute, Foreign Large-size Research Facility Application Supporting project, the Global Science Experimental Data Hub Center of the Korea Institute of Science and Technology Information and KREONET/ GLORIAD; the Polish Ministry of Science and Higher Education and the National Science Center; the Ministry of 
Science and Higher Education of the Russian Federation, Agreement 14.W03.31.0026; from 15.02.2018; the Slovenian Research Agency; Ikerbasque, Basque Foundation for Science, Spain; the Swiss National
Science Foundation; the Ministry of Education and the Ministry of Science and Technology of Taiwan; and the United States Department of Energy and the National Science Foundation.
[1] G. Pakhlova et al. (Belle Collaboration), Phys. Rev. D 77, 011103 (2008).

[2] G. Pakhlova et al. (Belle Collaboration), Phys. Rev. Lett. 98, 092001 (2007).

[3] G. Pakhlova et al. (Belle Collaboration), Phys. Rev. Lett. 100, 062001 (2008).

[4] G. Pakhlova et al. (Belle Collaboration), Phys. Rev. D 80, 091101 (2009).

[5] G. Pakhlova et al. (Belle Collaboration), Phys. Rev. D 83, 011101(R) (2011).

[6] B. Aubert et al. (BABAR Collaboration), Phys. Rev. D 76, 111105 (2007).

[7] B. Aubert et al. (BABAR Collaboration), Phys. Rev. D 79, 092001 (2009).

[8] P. del Amo Sanchez et al. (BABAR Collaboration), Phys. Rev. D 82, 052004 (2010).

[9] D. Cronin-Hennessy et al. (CLEO Collaboration), Phys. Rev. D 80, 072001 (2009).

[10] M. Ablikim et al. (BESIII Collaboration), Phys. Rev. Lett. 120, 132001 (2018).

[11] B. Aubert et al. (BABAR Collaboration), Phys. Rev. Lett. 95, 142001 (2005).

[12] M. Ablikim et al. (BESIII Collaboration), Phys. Rev. Lett. 100, 102003 (2008).

[13] T. E. Coan et al. (CLEO Collaboration), Phys. Rev. Lett. 96, 162003 (2006).

[14] C. Z. Yuan et al. (Belle Collaboration), Phys. Rev. Lett. 99, 182004 (2007).

[15] Z. Q. Liu et al. (Belle Collaboration), Phys. Rev. Lett. 110, 252002 (2013).

[16] M. Ablikim et al. (BESIII Collaboration), Phys. Rev. Lett. 118, 092001 (2017).

[17] B. Aubert et al. (BABAR Collaboration), Phys. Rev. Lett. 98 , 212001 (2007).

[18] X. L. Wang et al. (Belle Collaboration), Phys. Rev. Lett. 99, 142002 (2007).

[19] M. Ablikim et al. (BESIII Collaboration), Phys. Rev. D 96, 032004 (2017).

[20] T. V. Uglov, Y. S. Kalashnikova, A. V. Nefediev, G. V. Pakhlova, and P. N. Pakhlov, JETP Lett. 105, 1 (2017).

[21] G. Pakhlova et al. (Belle Collaboration), Phys. Rev. Lett. 101, 172001 (2008).

[22] M. Ablikim et al. (BESIII Collaboration), Phys. Rev. Lett. 122, 102002 (2019).

[23] M. Karliner and J. L. Rosner, Nucl. Phys. A954, 365 (2016).
[24] Throughout this analysis, for any given mode, the corresponding charge-conjugate mode is implied.

[25] A. Abashian et al. (Belle Collaboration), Nucl. Instrum. Methods Phys. Res., Sect. A 479, 117 (2002); also see Section II in J. Brodzicka et al., Prog. Theor. Exp. Phys. 2012, 04D001 (2012).

[26] S. Kurokawa and E. Kikutani, Nucl. Instrum. Methods Phys. Res., Sect. A 499, 1 (2003), and other papers included in this Volume.

[27] T. Abe et al., Prog. Theor. Exp. Phys. 2013, 03A001 (2013) and references therein.

[28] G. Rodrigo, H. Czyż, J. H. Kühn, and M. Szopa, Eur. Phys. J. C 24, 71 (2002).

[29] M. Tanabashi et al. (Particle Data Group), Phys. Rev. D 98 , 030001 (2018).

[30] S. Jia et al. (Belle Collaboration), Phys. Rev. D 98, 092015 (2018).

[31] H. Ikeda et al. (Belle Collaboration), Nucl. Instrum. Methods Phys. Res., Sect. A 441, 401 (2000).

[32] The Novosibirsk function is defined as $f(x)=$ $\exp \left[-\frac{1}{2}\left(\ln ^{2}\left(1+\Lambda\left(x-x_{0}\right)\right) / \tau^{2}+\tau^{2}\right)\right]$ with $\Lambda=\sinh (\tau \sqrt{\ln 4}) /$ $(\sigma \sqrt{\ln 4})$. The parameters represent the mean $\left(x_{0}\right)$, the width $(\sigma)$ and the tail asymmetry $(\tau)$.

[33] See Supplemental Material at http://link.aps.org/ supplemental/10.1103/PhysRevD.100.111103, for the recoil mass spectra against the $\gamma_{\mathrm{ISR}} D_{s}^{+}$system in $e^{+} e^{-} \rightarrow D_{s}^{+} D_{s 1}(2536)^{-}\left(\rightarrow \bar{D}^{* 0} K^{-}\right)$and $e^{+} e^{-} \rightarrow$ $D_{s}^{+} D_{s 1}(2536)^{-}\left(\rightarrow D^{*-} K_{S}^{0}\right)$ and for a summary of the values for the effective luminosity, the total reconstruction efficiency, the number of fitted signal events, and the product of the dressed cross section and the decay branching fraction $\sigma\left(e^{+} e^{-} \rightarrow D_{s}^{+} D_{s 1}(2536)^{-}\right) \times \mathcal{B}\left(D_{s 1}(2536)^{-} \rightarrow \bar{D}^{* 0} K^{-}\right)$in each $D_{s}^{+} D_{s 1}(2536)^{-}$mass bin.

[34] S. S. Wilks, Ann. Math. Stat. 9, 60 (1938).

[35] S. Godfrey and N. Isgur, Phys. Rev. D 32, 189 (1985).

[36] E. A. Kuraev and V.S. Fadin, Yad. Fiz. 41, 733 (1985) [Sov. J. Nucl. Phys. 41, 466 (1985)].

[37] X. Y. Gao, C. P. Shen, and C. Z. Yuan, Phys. Rev. D 95, 092007 (2017).

[38] The dressed cross section is $\sigma=\sigma^{B} /|1-\Pi|^{2}$, where $\sigma^{B}$ is the Born cross section, and $|1-\Pi|^{2}=0.94$ is the vacuum polarization factor [39].

[39] S. Actis et al., Eur. Phys. J. C 66, 585 (2010).

[40] N. Dash et al. (Belle Collaboration), Phys. Rev. Lett. 119, 171801 (2017). 